\title{
The evolved asymptotic giant branch stars in the central bar of the dwarf irregular galaxy NGC $6822^{\star, \star \star}$
}

\author{
A. Kang ${ }^{1,2}$, Y.-J. Sohn ${ }^{1,2}$, H.-I. Kim ${ }^{3}$, J. Rhee ${ }^{1,2}$, J.-W. Kim ${ }^{1,2}$, N. Hwang ${ }^{4}$, M. G. Lee ${ }^{4}$, Y.-C. Kim ${ }^{1}$, and M.-S. Chun ${ }^{1}$ \\ 1 Department of Astronomy, Yonsei University, Seoul 120-749, Korea \\ e-mail: sohnyj@yonsei.ac.kr; breeze82@galaxy.yonsei.ac.kr \\ 2 Center for Space Astrophysics, Yonsei University, Seoul 120-749, Korea \\ 3 Korea Astronomy and Space Science Institute, Daejeon 305-348, Korea \\ 4 Astronomy Division, Department of Physics and Astronomy, Seoul National University, Seoul 151-742, Korea
}

Received 23 December 2005 / Accepted 26 January 2006

ABSTRACT

\begin{abstract}
Images of $J H K$ and $g i$ obtained with the Canada-France-Hawaii Telescope are used to investigate the stellar contents of the resolved asymptotic giant branch (AGB) population in the area of $3.6 \times 66^{\prime} 3$ on the central bar of the dwarf irregular galaxy NGC 6822 . The upper envelope of near-infrared $(J-K, K)$ and $(H-K, K)$ color-magnitude diagrams (CMDs) shows four components of main-sequence stars, foreground stars, massive supergiants, and AGB stars. These components are also noticeable in the bright part of $(g-i, g)$ CMD. We select AGB stars in NGC 6822 from the $(g-K, g)$ CMD, which has a long spectral wavelength base line in the color index. There are $141 \mathrm{C}$ stars with a mean absolute magnitude of $\left\langle M_{K}\right\rangle=-7.60 \pm 0.50$, and with mean colors of $\left\langle(J-K)_{0}\right\rangle=1.77 \pm 0.41$, and $\left\langle(H-K)_{0}\right\rangle=0.75 \pm 0.28$. The number ratio of $\mathrm{C}$ stars to M-giants $(\mathrm{C} / \mathrm{M})$ is estimated as $0.27 \pm 0.03$, while the northern part of the bar has a somewhat lower C/M ratio than the southern part. The $(J-K)$ and $(H-K)$ color distributions of AGB stars contain a main peak of M-giant stars and a red tail of C stars. The broad color distributions for M-giants indicate that NGC 6822 has a wide range of intermediate ages with a peak at $\log \left(t_{\mathrm{yr}}\right) \sim 9$.0. The $M_{K}$ luminosity function (LF) for C stars in the central bar of NGC 6822 is likely to be a Gaussian distribution, while the LF of $\mathrm{C}$ stars in the southern part is slightly skewed to the brighter side than in northern part. The mean bolometric magnitude of $141 \mathrm{C}$ stars in NGC 6822 is $\left\langle M_{\mathrm{bol}}\right\rangle=-4.36 \pm 0.54$. The bolometric LF for M-giants extends up to $M_{\mathrm{bol}}=-6.5$, while the one for C stars spans $-5.8<M_{\mathrm{bol}}<-3.5$.
\end{abstract}

Key words. galaxies: individual: NGC 6822 - galaxies: stellar content - stars: AGB and post-AGB

\section{Introduction}

Dwarf galaxies are the most numerous galactic systems in the universe and are a major constituent of galaxy groups and clusters. A study of the stellar population in dwarf galaxies provides clues not only to stellar evolution and star formation history in the galaxies but also to the formation and evolutionary conditions of the galaxies and their parent galaxy clusters, hence to the universe itself. However, due to the low luminosity and small size of the systems, photometric studies of the resolved stars in dwarf galaxies are possible only for those in the Local Group (LG). Among bright resolved stars in LG dwarf galaxies, asymptotic giant branch (AGB) stars constitute the brightest intermediate-age stellar population consisting of oxygen-rich $\mathrm{M}$-giants and carbon-rich $\mathrm{C}$ stars with low to intermediate mass $\left(\sim 0.8-8 M_{\odot}\right)$. Therefore photometric studies of the AGB stars in dwarf galaxies provide clues to star formation in a galaxy for the intermediate age of $1 \sim 10$ Gyr (Tolstoy 2003; Grebel 1999) and offer an independent means of checking

* Based on observations carried out at the Canada-France-Hawaii Telescope, operated by the National Research Council of Canada, the Centre National de la Recherche Scientifique de France, and the University of Hawaii.

$\star \star$ Full Tables 2 and 3 are only available in electronic form at the CDS via anonymous ftp to

cdsarc.u-strasbg.fr $(130.79 .128 .5)$ or via

http://cdsweb.u-strasbg.fr/cgi-bin/qcat?J/A+A/454/717 the late evolution of stars at the post core-helium burning stage (e.g. Girardi et al. 2002).

Population studies of LG dwarf galaxies in the optical bands for bright resolved stars including AGB stars (e.g. Holtzman et al. 2002; Rizzi et al. 2003), taking advantage of deep and high angular resolution photometry from the Hubble Space Telescope (HST) and other ground-based telescopes, have revealed a complex star formation history in the galaxies (see, Grebel 20051997; van den Bergh 2000, 1999, for a review). Meanwhile, AGB stars appear quite prominently as late type bright giants in the near-infrared color-magnitude diagrams (CMDs) of LG dwarf galaxy (Davidge 2003a), because they are among the coolest bright stars of an intermediate-age stellar population. Moreover, the extreme red AGB stars, i.e. C stars, can be distinguished properly from M-giant AGB stars in the broadband near-infrared CMDs of nearby LG dwarf galaxies (e.g. Cioni \& Habing 2005; Davidge 2005; Kang et al. 2005; Sohn et al. 2006).

This paper is one of a series of papers studying the photometric properties of the AGB population and C stars in LG dwarf galaxies from near-infrared $J H K^{\prime}$ photometry, focused on the dwarf irregular galaxy NGC 6822. In previous papers (Kang et al. 2005; Sohn et al. 2006), we presented the nearinfrared photometric properties of AGB stars in dwarf elliptical galaxies NGC 185 and NGC 147, and demonstrated that it is possible to distinguish $\mathrm{C}$ stars from AGB stars using the near-infrared regime in $J H K^{\prime}$. We provided near-infrared photometric properties of 73 and $91 \mathrm{C}$ stars in NGC 185 and 
NGC 147, respectively, suggesting a wide range of age span for the intermediate-age stars in the galaxies. Note that NGC 6822 is a Magellanic type dwarf irregular galaxy, containing stellar populations of currently on going star formation in contrast to the dwarf ellipticals (Grebel 2005). The contamination of bright young stars makes it difficult to properly distinguish the AGB stars in the near-infrared CMDs of NGC 6822. Combining data from optical and near-infrared photometry allows one to investigate the dependence of stellar properties on color much better than simply relying on near-infrared or optical data alone (Momany et al. 2003; Ferraro et al. 2000; Guarnieri et al. 1998; Kuchinski et al. 1995; Cohen et al. 1978). Photometric parameters with a wide wavelength baseline through the optical to infrared can provide a reliable way to select the AGB population of $\mathrm{M}$-giants and $\mathrm{C}$ stars in NGC 6822. For this purpose, we use the $g^{\prime} i^{\prime}$ photometric data of NGC 6822 for the same area observed with $J H K^{\prime}$ bands.

NGC 6822 is not associated with any of the subgroups in the LG but is considered to be a member of the Local Group Cloud (Mateo 1998). As the nearest isolated dwarf galaxy, NGC 6822 is an important laboratory both for probing the evolution of a galaxy in isolated environments with minimal interaction and for a better understanding of star formation in this type of system. The global structure of NGC 6822 was surveyed first by Hodge (1977) and then in more detail by Hodge et al. (1991), tracing the galaxy to $10^{\prime}$ from its center through the central bar and to the surrounding outer circular structure. NGC 6822 consists of a relatively small optical bar $\left(6^{\prime} \times 11^{\prime}\right)$ oriented nearly north-south containing most of the sites of current star formation and an extended stellar halo surrounding the bar (Demers et al. 2005; Lee \& Hwang 2005). Also, NGC 6822 is unique among LG dwarf galaxies by having a much larger $\left(42^{\prime} \times 19^{\prime}\right)$ HI envelope than its optical bar (Weldrake et al. 2003; de Blok \& Walter 2000; Gottesman \& Weliachew 1977; Roberts 1972; Volders \& Högbom 1961).

A large number of population studies have investigated the stellar content and star-formation history of NGC 6822 and found constituent stars spanning a broad range of ages. Synthetic analysis (i.e. Wyder 2001; Gallart et al. 1996a) has shown that NGC 6822 most likely began forming stars at a very early epoch (12-15 Gyr ago) from low metallicity gas. The presence of evolved stars such as AGB and C stars and their spatial distribution (Cioni \& Habing 2005; Letarte et al. 2002; Gallart et al. 1994; Cook et al. 1986) suggest a high star-formation rate during the period 1-9 Gyr over a large area through NGC 6822, while synthetic analyses show a drop in star forming activity during the period of 3-5 Gyr ago (Wyder 2001; Gallart et al. 1996a). The rate of star formation began to increase again $~ 3 \mathrm{Gyr}$ ago (Tolstoy et al. 2001), presumably triggered by tidal interaction (de Blok \& Walter 2000; Hutchings et al. 1999), and it kept increasing over the last 100-200 Myr (Gallart et al. 1996b). NGC 6822 is forming stars actively at present, as shown by numerous HII regions (O’Dell et al. 1999; Hodge et al. 1988) and OB associations (Wilson 1992a,b), while blue stars having ages less than $\sim 600 \mathrm{Myr}$ and tracing the distribution of $\mathrm{HI}$ gas (Komiyama et al. 2003) are not yet very well mixed through the galaxy (Wyder 2001).

Several observations have been devoted to investigate the stellar population of resolved AGB and C stars over a large area of NGC 6822. Cook et al. (1986) developed a photometric technique using $\mathrm{TiO}$ and $\mathrm{CN}$ narrow bands to separate $\mathrm{C}$ stars from M-giants, and detected a significant population of C stars at regions in the central bar of NGC 6822. Letarte et al. (2002) applied the four-band photometric technique proposed by
Cook et al. (1986) to identify C stars and found $904 \mathrm{C}$ stars in $42^{\prime} \times 28^{\prime}$ fields centered on NGC 6822 , reporting the ratio of $\mathrm{C}$ stars to $\mathrm{M}$-giant stars $(\mathrm{C} / \mathrm{M})$ as $1.0 \pm 0.2$. They also found that the C stars of NGC 6822 extend over a much larger volume than the galaxy's optical image and exists in a spheroidal halo not restricted to the HI disk. Cioni \& Habing (2005) have detected many red-giant and AGB stars from the $I J K_{\mathrm{s}}$ images of the central $20^{\prime} \times 20^{\prime}$ of NGC 6822 and found a large spatial variation in metallicity of 1.56 dex from the estimated local $\mathrm{C} / \mathrm{M}$ values. That is twice as large as the spread found within Magellanic Clouds.

In this paper, we investigate the bright AGB stellar contents in the central bar of the nearby dwarf irregular galaxy NGC 6822 through optical to near-infrared photometry. Section 2 presents $g^{\prime} i^{\prime}$ and $J H K^{\prime}$ observations of NGC 6822, the data reduction procedure, and photometric measurements of the resolved stars. Section 3 presents the CMDs and color-color diagrams of the resolved stars based on $g^{\prime} i^{\prime}$ and $J H K^{\prime}$ photometry, from which we distinguish AGB stars from young blue objects in NGC 6822 and foreground stars. In Sect. 4, we discuss the near-infrared photometric properties of the identified $\mathrm{C}$ stars, along with the color distributions and luminosity functions of AGB stars. A summary of the results is given in Sect. 5.

\section{Observations, data reduction, and photometric measurements}

The observations were obtained with the $3.6 \mathrm{~m}$ Canada-FranceHawaii Telescope (CFHT) at Mouna Kea during two different runs in June 2004 and August 2003. The data in the $J H K^{\prime}$ bands were obtained using the CFHTIR imager on June 5, 2004. The CFHTIR is a near-infrared imager for use at the $\mathrm{f} / 8$ Cassegrain focus of the CFHT. It uses a $1024 \times 1024 \mathrm{Hg}$ :Cd:Te array with an angular scale of $0.211 /$ pixel, so that each image covers a total field-of-view of 3.6 6 3.'6 on the sky. Two overlapped fields were observed in the near-infrared $J H K^{\prime}$ with CFHTIR so as to cover a total area of $3.6 \times 6.3$ on the central bar of NGC 6822 . The total exposure time for each $J H K^{\prime}$ filter was $240 \mathrm{~s}$ for the complete observing sequence of eight $30 \mathrm{~s}$ exposures with a fourpoint square dither pattern. The MegaPrime imager mounted on the prime focus of the CFHT was used to observe in $g^{\prime} i^{\prime}$ bands on August 24, 2003. MegaPrime is a mosaic wide-field camera of $362048 \times 4612$ pixel CCD chips covering a full 1-degree fieldof-view with a resolution of 0 !' $185 /$ pixel. The total exposure times in $g^{\prime}$ and $i^{\prime}$ were 2100 and $1200 \mathrm{~s}$, respectively. While the original purpose of the $g^{\prime} i^{\prime}$ observation with MegaPrime was to search for the extended stellar halo of NGC 6822 (Hwang et al. 2005; Lee \& Hwang 2005), we used only two 6.4 $\times 14.4$ fields of the MegaPrime chip CCD14 and CCD23, in which the observed fields of the CFHTIR in $J H K^{\prime}$ were imbedded. A gap between the two MegaPrime chips leaves a gap of $13^{\prime \prime}$ in the two $g^{\prime} i^{\prime}$ band images. Figure 1 shows the observed fields, while the summary of the runs is presented in Table 1.

The $J H K^{\prime}$ data were reduced using the sequence: (1) subtraction of a dark frame, (2) division by dome flats, (3) subtraction of DC sky level, which was estimated by taking the mode of pixel intensity distribution in each flat-fielded frame, and (4) subtraction of the thermal signatures, which were constructed from median combined flat-fielded images of blank sky regions. The processed images were aligned to correct the dither offsets, then median-combined, and cropped to the region having a full $240 \mathrm{~s}$ exposure time. The seeings, as measured from the reduced images, are below $0 ! 7 F W H M$ in $J, H$, and $K^{\prime}$ images. Note that the central wavelength of $2.12 \mu \mathrm{m}$ 


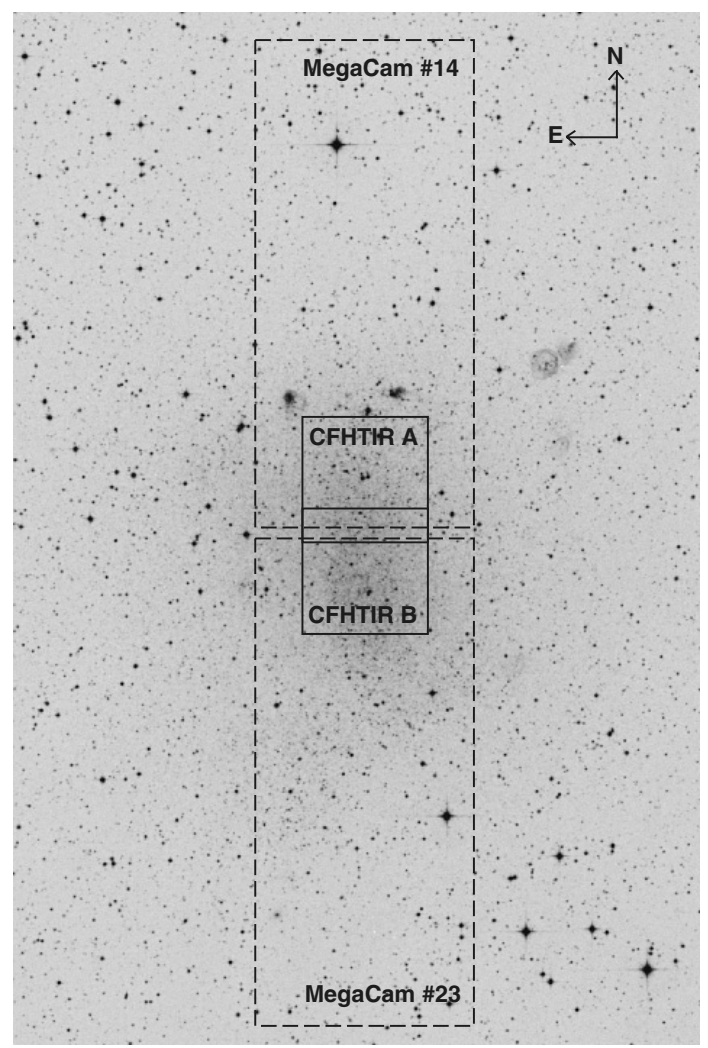

Fig. 1. The Digitized Sky Survey image of NGC 6822. The entire fieldof-view is $20^{\prime} \times 30^{\prime}$ with north up and east to the left. Two CFHTIR fields and two CCD14/CCD23 fields of MegaPrime are shown with boxes of solid and dashed lines, respectively. The two overlapped CFHTIR fields cover an area of 3.6 6 6.3 on central bar NGC 6822. Two MegaPrime fields cover 6. $4 \times 14^{\prime} .4$ each, in which the CFHTIR field is imbedded.

Table 1. Observational log.

\begin{tabular}{cccc}
\hline \hline Filter & Exp. time $(\mathrm{s})$ & $F W H M\left({ }^{\prime \prime}\right)$ & Date \\
\hline$g^{\prime}$ & 2,100 & 1.07 & Aug. 24, 2003 \\
$i^{\prime}$ & 1,200 & 0.75 & " \\
$J$ & $8 \times 30$ & 0.60 & Jun. 5, 2004 \\
$H$ & $8 \times 30$ & 0.67 & " \\
$K^{\prime}$ & $8 \times 30$ & 0.66 & " \\
\hline
\end{tabular}

for the $K^{\prime}$ filter of CFHTIR is slightly shorter than those of the standard $K(2.20 \mu \mathrm{m})$ and $K_{\mathrm{S}}(2.15 \mu \mathrm{m})$ filters. This leads to a lower thermal component of sky background in $K^{\prime}$ and deeper imaging with the same integration time as in the standard $K$ and $K_{S}$ filters (Simons \& Tokunaga 2002; Tokunaga et al. 2002; Wainscoat \& Cowie 1992). The $K^{\prime}$ filter shows a zero point approximately $0.03 \sim 0.04 \mathrm{mag}$ fainter than the zero point for standard $K$ filter (Wainscoat \& Cowie 1992), indicating that the difference does not seriously affect the limits of magnitude and colors for the definition of AGB stellar populations in the current study. Preprocessing of the raw $g^{\prime} i^{\prime}$ data was done using the Elixir, which is an extensive data assessment, calibration, and preprocessing system for the MegaPrime observations of the CFHT. The seeings in each $g^{\prime}$ and $i^{\prime}$ image reduced by the Elixir preprocessing pipeline are 1.'07 and 0.75 FWHM, respectively. Hereafter, $K^{\prime}$ filter is simply referred to as $K$, and $g^{\prime} i^{\prime}$ refer to $g i$ as well.

The point-spread-function fitting routines DAOPHOT II/ ALLSTAR (Stetson \& Harris 1988; Stetson 1987) and ALLFRAME (Stetson 1994) were used to measure the

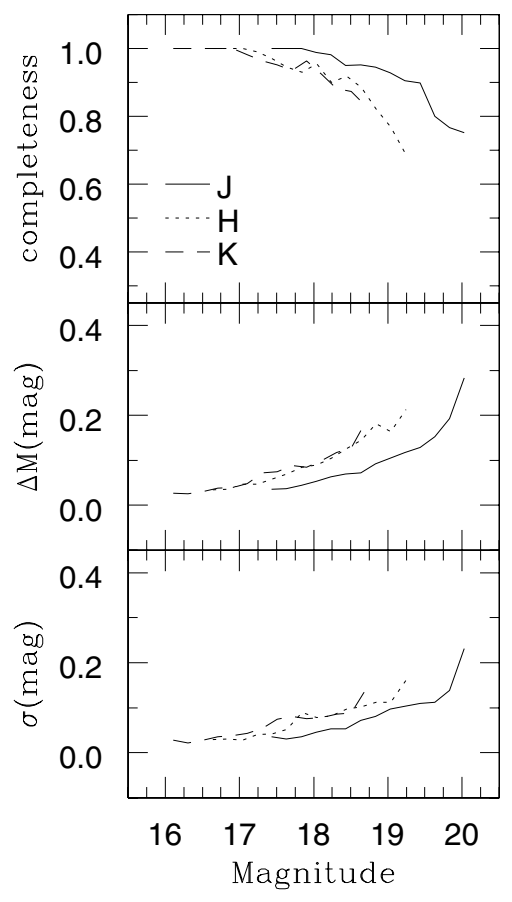

Fig. 2. The results of artificial star experiments. Solid, dotted, and dashed lines represent $J, H$, and $K$ bands. The completeness is the number of recovered artificial stars divided by the total number of stars added. $\Delta M$ (mag) is the mean difference between the actual input magnitudes and the measured magnitudes by DAOPHOTII/ALLSTARS, and $\sigma$ (mag) is the standard deviation of the $\Delta M$.

brightness of individual objects in NGC 6822. The instrumental magnitudes of $J H K$ from CFHTIR were calibrated by observations of UKIRT faint standard stars, and those of $g i$ from MegaPrime were transformed into the standard system using transformation relations provided by the CFHT staff of the queue observations. A total of 12611 stars were detected in $J H K$ filters on the central 3.' $6 \times 6.3$ field of the CFHTIR observations, while a total of 32983 stars were detected in the CFHTIR field on $g i$ images of the two 6!4 $\times 14 ! .4$ fields of the MegaPrime observations. The positions of several bright stars were compared to match stars measured in all five band images, for which the analyzed area is restricted to the CFHTIR field, i.e. 3. $6 \times 6.3$ field-of-view of the central bar in NGC 6822, except for the interim gap induced from two MegaPrime chips. Finally, a total of 10029 stars were commonly detected in all five giJHK images.

To accomplish the main purpose of this paper, i.e. studying the near-infrared photometric properties for the M-giant and C stars of AGB population in NGC 6822, artificial star experiments on the $J H K$ images were run to determine completeness fractions and uncertainties in the photometric measurements. Only a hundred artificial stars were added in each experiment to prevent significant increases in the amount of crowding. We did 15 experiments in each $J H K$ image of the two fields observed with the CFHTIR so as to add 1500 artificial stars in each frame. The estimated completeness fractions were not significantly different in each field, and Fig. 2 shows the averaged results for each $J H K$ image. In Fig. 2, completeness is the recovery rate of the input artificial stars, $\Delta M$ is the rms difference in magnitudes between the input and the measured brightness values, and $\sigma$ is the standard deviation of $\Delta M$. When $J, H$, and $K$ are fainter than $19.2,18.7$, and $18.3 \mathrm{mag}$, respectively, the completeness of each band is less than $\sim 90 \%$. This indicates that the AGB stars with magnitude brighter than the assumed tip of 


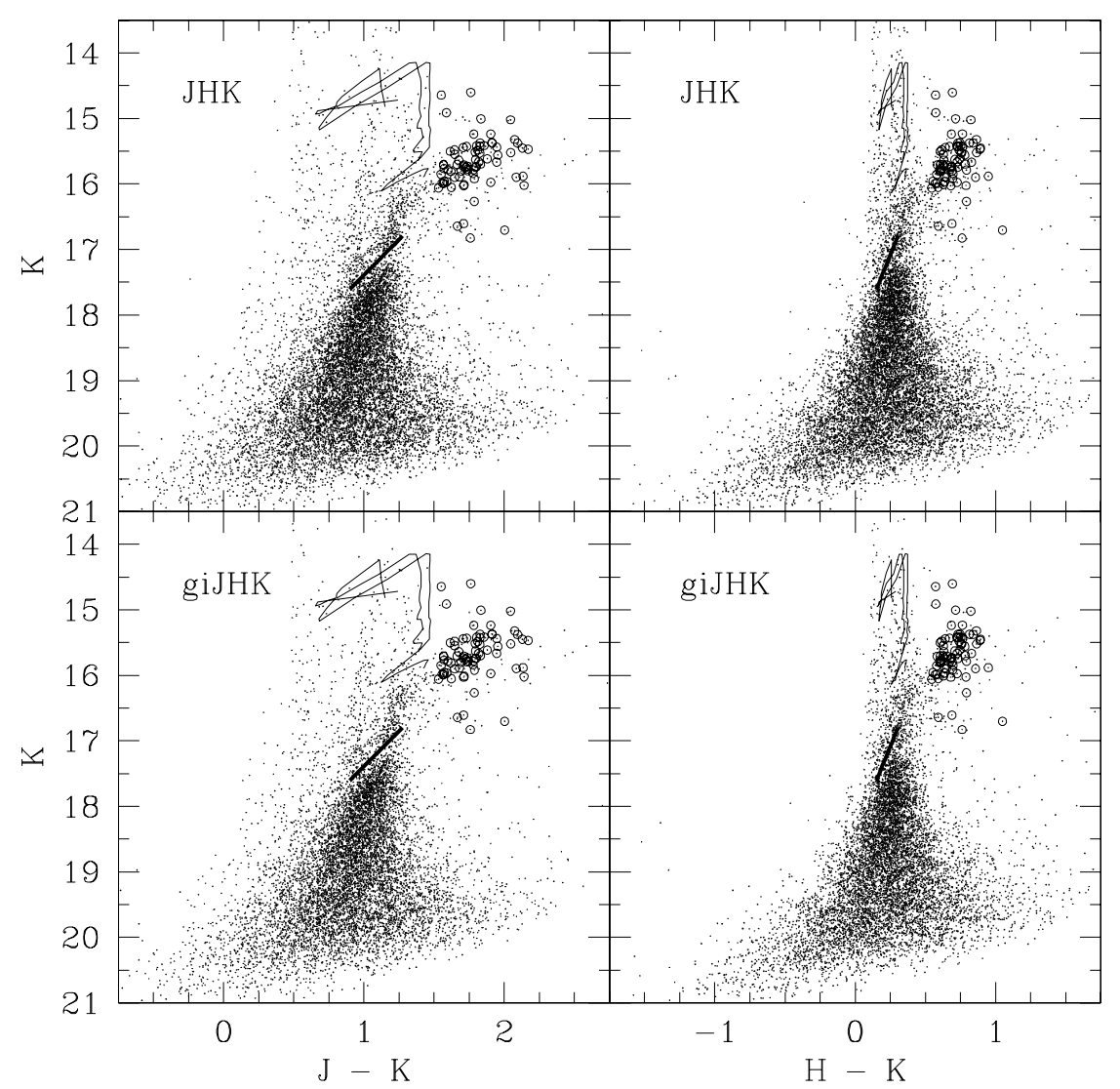

Fig. 3. $(J-K, K)$ and $(H-K, K)$ CMDs for stars detected in near-infrared $J H K$ bands (upper) and commonly in all five giJHK bands (lower) at the observed 3'. $6 \times 6$ ' 3 field of NGC 6822. The loci with thin lines trace the photometric evolution of the AGB tip in the range of $\log \left(t_{\mathrm{yr}}\right)=7.8 \sim 10.2$ with $Z=0.004$ and 0.008 , respectively (Girardi et al. 2002). The estimated color and magnitude range of the RGB tip at the distance of NGC 6822 also is presented, but with a thick line. Open circles indicate $72 \mathrm{C}$ stars cross-identified in both giJHK photometry (this paper) and TiO/CN band photometry (Letarte et al. 2002).

red-giant branch (TRGB), i.e. $K=17.20$ (Sect. 3.2), are not affected by the crowding effect in the observed images.

\section{Photometric properties of NGC 6822}

\subsection{Adopted reddening, distance and metallicity range}

From the $U B V$ photometry of OB stars, Massey et al. (1995) derived an enormous spread in the local color excess with $E(B-V)=0.26$ in the outer eastern/western parts and $E(B-V)=$ 0.45 in the vicinity of the bar of NGC 6822. Cioni \& Habing (2005) noticed, however, that the significant amount of reddening in the central region may be due to dust obscuring young objects and hardly affects the near-infrared photometry of redgiant branch (RGB) or AGB stars. From the dust infrared emission feature on the COBE and IRAS maps of $100 \mu \mathrm{m}$, Schlegel et al. (1998) obtained the reddening $E(B-V)=0.24$. Adopting this value and applying the reddening ratios of Schlegel et al. (1998), we estimated the interstellar absorptions in $J H K$ bands to be $A_{J}=0.216, A_{H}=0.138$ and $A_{K}=0.088$, and then the reddening values of $E(J-K)=0.128$ and $E(H-K)=0.050$.

The distance modulus of NGC 6822 has been measured directly using the period-luminosity $(P-L)$ relation of Cepheids and the magnitude of the TRGB. McAlary et al. (1983) estimated the distance modulus of NGC 6822 to be $(m-M)_{0}=23.47 \pm 0.11$ from the near-infrared $H$ band photometry of 9 Cepheids in NGC 6822. Visvanathan (1989) estimated $(m-M)_{0} \sim 23.2$ from infrared observations of 3 Cepheids in NGC 6822. Using $I$ magnitude of TRGB, Lee et al. (1993) and Gallart et al. (1996c) obtained the distance modulus of $23.46 \pm 0.10$ and $23.4 \pm 0.10$, respectively. Gallart et al. (1996c) also obtained the distance modulus of $(m-M)_{0}=23.49 \pm 0.08$ using the optical $B V R I$ band photometry of 6 Cepheids in NGC 6822. Cioni \& Habing (2005) measured the distance modulus of $(m-M)_{0}=23.34 \pm 0.12$ using the $I$ magnitude of TRGB. Pietrzyński et al. (2004) measured the distance modulus to be $(m-M)_{0}=23.34 \pm 0.04$ using the $P-L$ relation of 21 Cepheids in NGC 6822 . We have adopted this value.

Cohen \& Blakeslee (1998) obtained the spectra of two bright clusters in NGC 6822, i.e. cluster VII, considered a genuine old globular cluster, and cluster VI, considered a younger cluster. They showed that the metallicity of cluster VII is $[\mathrm{Fe} / \mathrm{H}]=$ $-1.95 \pm 0.15$, which corresponds to that of typical metal-poor globular cluster, and that of cluster VI is $[\mathrm{Fe} / \mathrm{H}] \approx-1.0$, which is comparable to the present abundance of the interstellar medium in NGC 6822. Using high-resolution spectra of two young A-type supergiants near the center of NGC 6822, Venn et al. (2001) obtained the mean metallicity of $\langle[\mathrm{Fe} / \mathrm{H}]\rangle=-0.49 \pm$ 0.22 . From the spectroscopic metallicity distribution functions for RGB stars, Tolstoy et al. (2001) found that RGB stars in NGC 6822 field showed a mean metallicity of $[\mathrm{Fe} / \mathrm{H}]=-1.0 \pm$ 0.5 with a significant spread in the range of $-2.0<[\mathrm{Fe} / \mathrm{H}]<$ -0.5 . From the slope of the RGB on the $(J-K, K)$ CMDs, Davidge (2003b) determined the metallicity of the RGB in the disk of $\mathrm{NGC} 6822$ as $[\mathrm{Fe} / \mathrm{H}]=-1.3 \pm 0.3$. These previous measurements indicate that NGC 6822 has a wide spread of metallicity in the range of $[\mathrm{Fe} / \mathrm{H}]=-2.0 \sim-0.5$. 


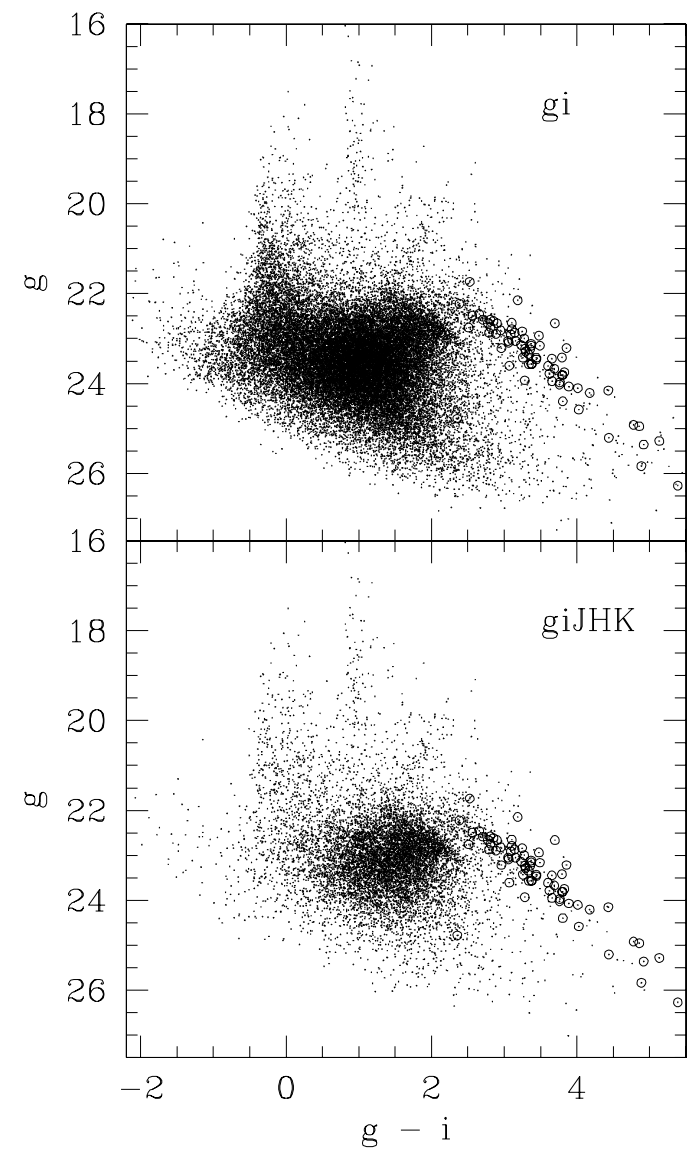

Fig. 4. $(g-i, g)$ CMDs for stars detected in gi bands (upper) and commonly in all five giJHK bands (lower). Open circles are the same as in Fig. 3.

\subsection{Color-magnitude diagrams}

Figures 3 and 4 show near-infrared $(J-K, K)$ and $(H-$ $K, K)$ CMDs, and $(g-i, g)$ CMD of the resolved stars in the observed 3.6 $6 \times 6 ! 3$ area of NGC 6822. The upper diagrams in Figs. 3 and 4 contain stars detected only in $J H K$ bands and gi bands, respectively, while the lower diagrams are CMDs of stars detected commonly in all five giJHK bands. The loci with thin line in Fig. 3 indicate the photometric evolution of the AGB tips (Girardi et al. 2002) in the range of $\log \left(t_{\mathrm{yr}}\right)=7.8 \sim 10.2$ with $Z=0.004$ and 0.008 at the adopted distance of NGC 6822 . Using the empirical relations between the TRGB brightness in near-infrared bands and metallicity for stars in a galaxy (Valenti et al. 2004), we estimated the range of magnitude and color of the TRGB for the given metallicity range $[\mathrm{Fe} / \mathrm{H}]=-2.0 \sim-0.5$ (Sect. 3.1) of NGC 6822. The thick lines of $16.80<K<$ $17.61,0.90<(J-K)<1.28$, and $0.10<(H-K)<0.25$ in Fig. 3 are the calculated loci of the TRGB at the adopted distance and metallicity range of NGC 6822 . In this paper, we consider that AGB stars are located above the mean magnitude $K=17.20$ of the TRGB extending up to the brightness of the AGB tip, even though a few RGB stars can be located at $K<17.2$ because the $K$ magnitude of the TRGB depends on the $(J-K)$ color (cf. Cioni \& Habing 2005).

The upper envelope of near-infrared $(J-K, K)$ CMD in Fig. 3 shows four different components: (1) a faint blue component with $J-K \sim 0.3$ from main-sequence stars in NGC 6822; (2) a bright blue component from Galactic foreground stars with $0.4<J-K<0.8$; (3) a vertical yellow sequence from massive supergiant stars with $J-K \sim 1.1$; and (4) an extended bright red plume from M-giant and C stars of AGB with $J-K>1.1$. These components are also found on the bright part of the $(g-i, g)$ CMD in Fig. 4, corresponding to three vertical branches and one red tail. The bluest component with $g-i \sim 0$ consists of massive main-sequence stars in NGC 6822 (Wyder 2001; Gallart et al. 1996c), and the vertical blue sequence with $g-i \sim 1$ corresponds to the foreground Galactic stars (Cioni \& Habing 2005; Gallart et al. 1996c). The extended yellow branch of $1.7 \lesssim g-i \lesssim 2.2$ contains massive supergiants in NGC 6822 (Gallart et al. 1996c, 1994). The AGB population of M-giants and C stars in NGC 6822 is imbedded in the red tail shown in the $(g-i, g)$ CMD. This feature is clearer in the lower panel of Fig. 4, which is $(g-i, g)$ CMD for stars detected in all five giJHK bands. This is because the faint blue stars are hardly detected in the near-infrared wavelength, whereas it is easy to detect the red AGB stars.

We note that the Galactic star count model of Ratnatunga \& Bahcall (1985) predicted 210 bright blue foreground stars with $(B-V)<1.3$ and $V<22$ in the field-of-view of the CFHTIR observation, which is consistent with the rough count of bright foreground stars in Figs. 3 and 4. For the detailed AGB population study, the two other bright components of the massive supergiants and the blue main-sequence stars should also be separated and removed properly from the bright part of the obtained CMDs.

\subsection{Selection of $A G B$ stars and $C$ stars}

As noted in Sect. 1, cool AGB stars can be properly distinguished by taking advantage of a wide wavelength baseline photometry. Figure 5 shows CMDs of stars with color indices of $g i$ minus $J H K$ bands and in $g i$ magnitudes. All painted points, except for the black ones, indicate stars having $K$ magnitude brighter than the assigned TRGB magnitude of $K=17.2$ (Sect. 3.2), which corresponds to the slanted lines in $(i-K, i)$ and $(g-K, g)$ CMDs in Fig. 5. As can be seen in Fig. 5, the longer the wavelength range of the color index, the wider the spread of color and magnitude of bright stars. Indeed, three classes of bright objects such as AGB stars, supergiant stars and Galactic foreground stars, are differentiated most remarkably in $(g-K, g) \mathrm{CMD}$, which has the longest spectral wavelength base line of color index among the CMDs in Fig. 5. From a few empirical tests by eye-fit of the tangential to the plume of AGB stars in $(g-K, g) \mathrm{CMD}$, we determined the border line of constant magnitude of $g=21.5$ to separate AGB and supergiant stars. Sources located above the TRGB in $K$ and $g \geq 21.5$ are assigned as AGB candidates, and a total of 663 stars are then selected for AGB stars. The bright Galactic foreground stars in Fig. 5 have the infrared color range of $0.4<J-K<0.8$ (Sect. 3.2). Supergiant stars in NGC 6822 are well separated from AGB stars and foreground stars in $(g-K, g)$ CMD of Fig. 5. Note that it is difficult to distinguish supergiant stars from AGB stars in CMDs with a short wavelength base line of color index (e.g. Cioni et al. 2000a), because supergiants generally have spectral type M or K and usually are located in positions similar to AGB stars. The classified AGB, supergiant and foreground stars are plotted in the other CMDs of Fig. 5 with same colors in $(g-K, g)$ CMD. The bulk of RGB stars, the oldest population in NGC 6822 , occurs below the TRGB, and main-sequence stars occupy the bright blue part of $(g-K, g)$ CMD.

To confirm the population classification for the bright stars above, we compare the theoretical isochrones of AGB stars with the observed $(J-K, K)$ CMD in Fig. 6 for stars 

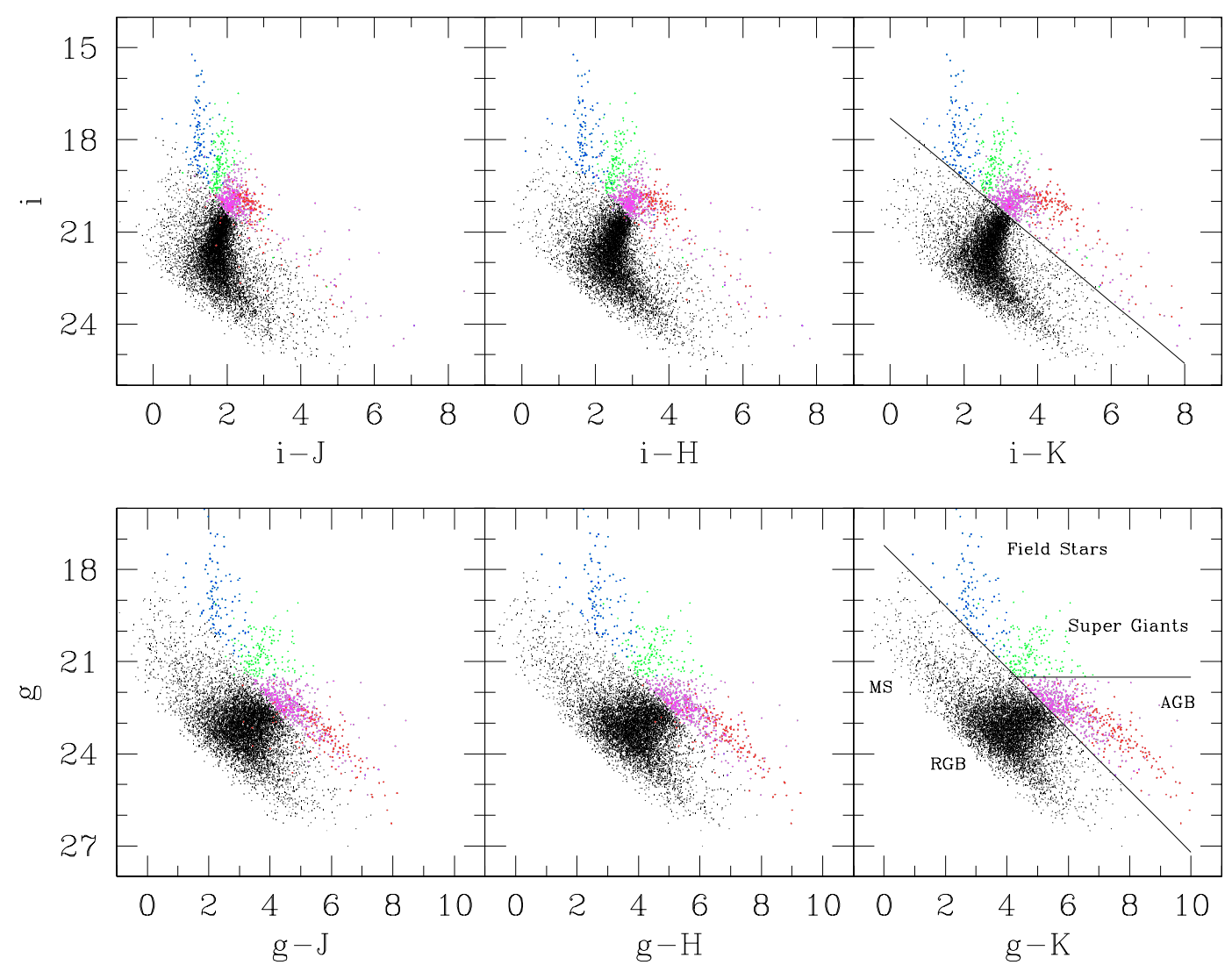

Fig. 5. CMDs of stars in the color indices of gi minus $J H K$ bands and in magnitudes of gi bands. All painted points except black ones indicate stars having brighter $K$ magnitude than the TRGB at $K=17.2$, which corresponds to the slanted lines in $(i-K, i)$ and $(g-K, g)$ CMDs. Blue and green points correspond to Galactic foreground stars and massive supergiants, respectively. AGB stars are represented by magenta points for M-giants and red points for $\mathrm{C}$ stars.

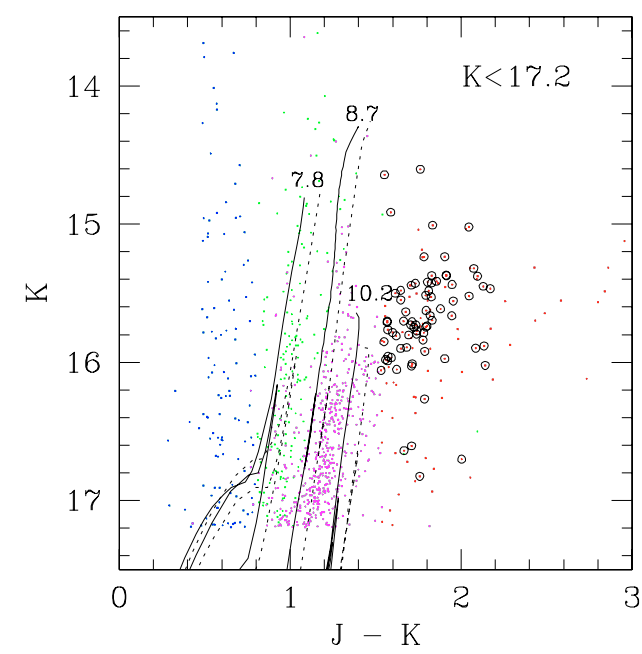

Fig. 6. The theoretical isochrones of $\log \left(t_{\mathrm{yr}}\right)=7.8,8.7$ and 10.2 with $Z=0.004$ (solid lines) and $Z=0.008$ (dotted lines) (Girardi et al. 2002) on the near-infrared $(J-K, K)$ CMD of stars brighter than $K=17.2$ in NGC 6822 . The colors of points are the same as in Fig. 5. Open circles are the same as in Fig. 3.

having $K$ magnitudes brighter than 17.2 of the assigned magnitude of TRGB. It is apparent in Fig. 6 that most of supergiant stars are clearly separated from AGB stars at $\log \left(t_{\mathrm{yr}}\right)<8.7$, i.e. younger than $\sim 500 \mathrm{Myr}$. Assuming the youngest age of $\log \left(t_{\mathrm{yr}}\right)=7.8$, supergiant stars are also clearly distinguished from the Galactic foreground stars. The distribution of most AGB stars between isochrones of $\log \left(t_{\mathrm{yr}}\right)=8.7$ and 10.2 suggests that the star formation in NGC 6822 had been continuous for the period of intermediate age.

The evolutionary mechanism of the AGB stage changes the atmospheric abundance of stars, starting with the oxygen-rich atmosphere of M-giant stars to carbon-rich C stars (Nowotny et al. 2001), which causes them to be located at different parts of the CMD and color-color diagram. Cioni et al. (2000b) found that the $(J-K, K) \mathrm{CMD}$ of AGB stars in Magellanic Clouds contain two sequences; one vertical sequence of O-rich stars with $M$ type spectra and a separate branch of C-rich stars that departs to redder colors from the vertical sequence. Color-color diagrams in near-infrared bands have been used as diagnostic tools to identify $\mathrm{C}$ star candidates from AGB stars (e.g. Davidge 2005; Kang et al. 2005; Sohn et al. 2006; Marigo et al. 2003; Wood et al. 1985; Frogel et al. 1980). Based on the previous results, Davidge (2005) assigned the color limits of $(J-K)_{0}>1.4$ and $(H-K)_{0}>0.45$ to separate C stars from M-giants of the AGB population in nearby dwarf galaxies. Adopting these values, we distinguish $\mathrm{C}$ stars from the bulk of the M-giant stars of the AGB population in NGC 6822 by using the color limits $(J-K)>1.53$ and $(H-K)>0.50$. Figure 7 shows the $(H-K)-(J-K)$ color-color diagram and the color histograms of selected AGB stars with the color criteria of C stars. It is apparent in Fig. 7 that $\mathrm{C}$ stars are clearly distinguished from M-giants in a color-color diagram, and the color distribution has a main peak of M-giants and a red tail of $\mathrm{C}$ stars. Finally, a total of $141 \mathrm{C}$ stars are selected from the color-color diagram and 
Table 2. Coordinates and photometric properties of $141 \mathrm{C}$ stars, and color indices in $R I$ bands of cross-identified $72 \mathrm{C}$ stars. Columns 14 and 15 list the $(R-I)$ colors and ID numbers of cross-identified C stars in Letarte et al. (2002). The full table is available in electronic form at the CDS.

\begin{tabular}{ccccccccccccccc}
\hline \hline ID & RA(J2000) & $\operatorname{Dec}(\mathrm{J} 2000)$ & $K$ & $\sigma_{K}$ & $J-K$ & $\sigma_{(J-K)}$ & $H-K$ & $\sigma_{(H-K)}$ & $g$ & $\sigma_{g}$ & $g-i$ & $\sigma_{(g-i)}$ & $(R-I)^{a}$ & $\mathrm{ID}_{L}{ }^{a}$ \\
\hline 1 & 194448.60 & -14493.5 & 16.022 & 0.021 & 2.141 & 0.035 & 0.790 & 0.025 & 23.466 & 0.042 & 3.443 & 0.046 & 1.679 & 277 \\
2 & 194448.92 & -144737.6 & 15.516 & 0.022 & 1.793 & 0.024 & 0.755 & 0.027 & 24.070 & 0.071 & 3.889 & 0.073 & 1.590 & 285 \\
3 & 194449.14 & -144950.8 & 15.007 & 0.014 & 1.833 & 0.023 & 0.716 & 0.018 & 23.215 & 0.039 & 3.857 & 0.043 & 1.610 & 293 \\
\hline
\end{tabular}

${ }^{a}$ Letarte et al. (2002).

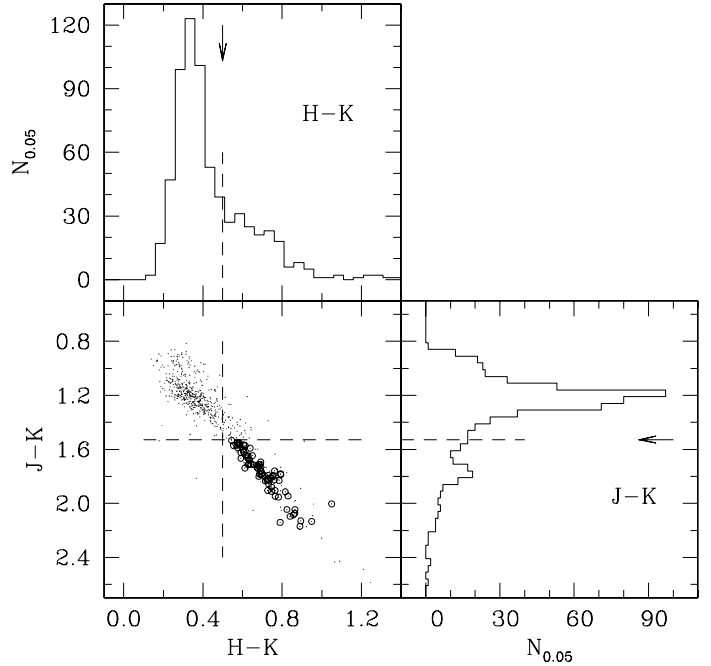

Fig. 7. $(H-K)-(J-K)$ color-color diagram and color histograms of $(H-K)$ (upper) and $(J-K)($ right) for selected AGB stars. The dashed lines and arrows represent the color criteria of $(J-K)=1.53$ and $(H-K)=0.05$ to separate $\mathrm{C}$ stars from M-giants of AGB stars. Open circles are the same as in Fig. 3.

color histograms for the observed 663 AGB stars of NGC 6822. The equatorial coordinates and the measured photometric properties of selected $\mathrm{C}$ stars are listed in Table 2. Table 3 lists those of the rest $522 \mathrm{M}$-giants of AGB stars.

\section{Discussion}

\section{1. $C$ stars and $C / M$ ratio}

The near-infrared survey of $\mathrm{C}$ stars in nearby galaxies can be obtained from moderately short exposures, and will provide an efficient means of identifying C stars from M-giant stars (Kang et al. 2005; Sohn et al. 2006; Marigo et al. 2003; Davidge 2003a). From the near-infrared color-color diagram of NGC 6822, we separated 141 red C stars from M-giant stars of the AGB population selected from $g i J H K$ photometry. The mean magnitude and colors of the $141 \mathrm{C}$ stars in the central bar of NGC 6822 are estimated to be $\langle K\rangle=15.82 \pm 0.50,\langle(J-K)\rangle=1.90 \pm 0.41$, and $\langle(H-K)\rangle=0.80 \pm 0.28$, which correspond to $\left\langle M_{K}\right\rangle=-7.60$, $\left\langle(J-K)_{0}\right\rangle=1.77$, and $\left\langle(H-K)_{0}\right\rangle=0.75$ with the adopted distance modulus and reddening values in Sect. 3.1.

To investigate the spatial dependence of the photometric properties of $\mathrm{C}$ stars in the bar of NGC 6822, we divided the observed CFHTIR field of 3.6 $\times 6.3$ area into two regions. As in Fig. 1, the 13" gap between the MegaPrime chips separates the CFHTIR field naturally into the northern 3.6x $3 ! 3$ region and southern $3.6 \times 2$. 9 region of the observed central bar area. Among $141 \mathrm{C}$ stars identified in the observed central bar region of NGC 6822, $53 \mathrm{C}$ stars are found in the northern part with $\langle K\rangle=15.89 \pm 0.55,\langle(J-K)\rangle=1.98 \pm 0.51$ and $\langle(H-K)\rangle=0.89 \pm 0.37$, while $88 \mathrm{C}$ stars are found in the southern part with $\langle K\rangle=15.78 \pm 0.46,\langle(J-K)\rangle=1.85 \pm 0.33$ and $\langle(H-K)\rangle=0.75 \pm 0.19$. The quoted uncertainties in the magnitude and colors are $1 \sigma$ standard errors in the mean. The mean magnitude and colors of $\mathrm{C}$ stars in the southern part are slightly brighter and bluer than those of $\mathrm{C}$ stars in the northern part with quite a small confidence level less than $1 \sigma$ standard errors of the mean.

Using the CFH12K camera, Letarte et al. (2002) identified $904 \mathrm{C}$ stars in $42^{\prime} \times 28^{\prime}$ area centered on NGC 6822 using the four-band photometric technique including two narrow bands of $\mathrm{CN} / \mathrm{TiO}$ and $R I$ bands. We cross-identified $\mathrm{C}$ stars detected in giJHK bands with $\mathrm{C}$ stars detected by Letarte et al. (2002). A total $72 \mathrm{C}$ stars are commonly matched. In Figs. 3, 4, 6, and 7 , the cross-identified $C$ stars are presented by open circles. Figure 8 shows color-color diagrams of $J H K$ and $R I$ bands for the cross-identified $\mathrm{C}$ stars. The color limit of $\mathrm{C}$ stars in $R I$ bands in Fig. 8 were adopted from Letarte et al. (2002). The ID and $(R-I)$ color of cross-identified $C$ stars are listed in Table 2 . The mean absolute magnitude and colors in $J H K$ for the 72 crossidentified $\mathrm{C}$ stars are estimated to be $\left\langle M_{K}\right\rangle=-7.74 \pm 0.40$, $\left\langle(J-K)_{0}\right\rangle=1.65 \pm 0.17$ and $\left\langle(H-K)_{0}\right\rangle=0.66 \pm 0.10$.

From the $\mathrm{C}$ star survey in LG galaxies, Battinelli \& Demers (2004b) found that the mean absolute magnitudes of C stars in the $I$ band are essentially identical for most of the nearby galaxies, while the $\left\langle M_{I}\right\rangle_{\mathrm{S}}$ of NGC 185 and NGC 147 are slightly fainter than those of other nearby galaxies (see also Nowotny et al. 2003). The currently estimated mean absolute magnitude $\left\langle M_{K}\right\rangle=-7.60$ of $141 \mathrm{C}$ stars in NGC 6822 seems to be comparable to that of $\mathrm{C}$ stars in the other nearby dwarf elliptical galaxies NGC 147 and NGC 185 (cf. Kang et al. 2005; Sohn et al. 2006), and colors in $(J-K)$ and $(H-K)$ for the $C$ stars as well. The $73 \mathrm{C}$ stars in NGC 185 have $\left\langle M_{K}\right\rangle=-7.93 \pm 0.38$, $\left\langle(J-K)_{0}\right\rangle=2.25 \pm 0.46$, and $\left\langle(H-K)_{0}\right\rangle=0.87 \pm 0.25$ (Kang et al. $2005)$, and the $91 \mathrm{C}$ stars in NGC 147 have $\left\langle M_{K}\right\rangle=-7.56 \pm 0.47$, $\left\langle(J-K)_{0}\right\rangle=1.81 \pm 0.46$, and $\left\langle(H-K)_{0}\right\rangle=0.74 \pm 0.31$ (Sohn et al. 2006). In order to confirm the identical mean absolute magnitudes of $\mathrm{C}$ stars in the near-infrared wavelengths, a more homogeneous data set is needed for the LG galaxies.

We estimate the number ratio of $\mathrm{C}$ stars to $\mathrm{M}$-giants $(\mathrm{C} / \mathrm{M})$ of the AGB population to be $0.27 \pm 0.03$ at the total observed area of the central bar of NGC $6822,0.22 \pm 0.03$ at the northern part, and $0.31 \pm 0.04$ at the southern part of the bar. The local C/M ratios at the central bar of NGC 6822 indicate that the northern part has a rather lower $\mathrm{C} / \mathrm{M}$ ratio than the southern part of the bar. From observations in the $J K_{\mathrm{s}}$ bands covering the central $20^{\prime} \times 20^{\prime}$ of NGC 6822 , Cioni \& Habing (2005) found $1511 \mathrm{C}$ stars and estimated the global $\mathrm{C} / \mathrm{M}$ ratio to be 0.32 . They also recognized that the distribution of the $\mathrm{C} / \mathrm{M}$ ratio is rather clumpy and the bar itself has a relatively low $\mathrm{C} / \mathrm{M}$ ratio in the northern part, just as we found.

A variation of the observed $\mathrm{C} / \mathrm{M}$ ratio in a galaxy has been explained by a variation in the metallicity. Iben \& Renzini (1983) explained that O-rich AGB stars, i.e. M-giants, easily become 
Table 3. Coordinates and photometric properties of $522 \mathrm{M}$-giant stars in NGC 6822. The full table is available in electronic form at the CDS.

\begin{tabular}{ccccccccccccc}
\hline \hline ID & RA(J2000) & $\operatorname{Dec}(\mathbf{J} 2000)$ & $K$ & $\sigma_{K}$ & $J-K$ & $\sigma_{(J-K)}$ & $H-K$ & $\sigma_{(H-K)}$ & $g$ & $\sigma_{g}$ & $g-i$ & $\sigma_{(g-i)}$ \\
\hline 1 & 194448.49 & -144856.7 & 17.181 & 0.035 & 0.944 & 0.047 & 0.243 & 0.047 & 24.024 & 0.065 & 0.738 & 0.110 \\
2 & 194448.57 & -14480.8 & 16.340 & 0.025 & 1.343 & 0.038 & 0.318 & 0.030 & 24.065 & 0.072 & -0.412 & 0.268 \\
3 & 194448.57 & -144945.9 & 16.894 & 0.020 & 1.077 & 0.033 & 0.238 & 0.027 & 23.579 & 0.051 & -0.197 & 0.146 \\
\hline
\end{tabular}

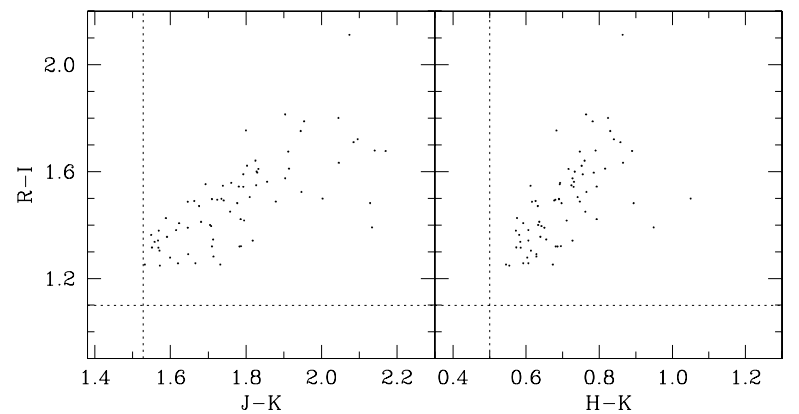

Fig. 8. Color-color diagrams of $J H K$ and $R I$ bands for 72 crossidentified $\mathrm{C}$ stars by Letarte et al. (2002). Vertical dotted lines with $(J-K)=1.53$ and $(H-K)=0.50$ are the color limits of $\mathrm{C}$ stars in $J H K$ photometry, and horizontal dotted lines with $(R-I)=1.1$ are the color limits of $C$ stars in RI photometry by Letarte et al. (2002).

Table 4. The number of $\mathrm{C}$ stars and M-giants, $\mathrm{C} / \mathrm{M}$ ratio, and metallicity in the central bar of NGC 6822 .

\begin{tabular}{cccccc}
\hline \hline & $\mathrm{C}$ & $\mathrm{M}$ & $\mathrm{C} / \mathrm{M}$ & {$[\mathrm{Fe} / \mathrm{H}]^{a}$} & {$[\mathrm{Fe} / \mathrm{H}]^{b}$} \\
\hline total & 141 & 522 & $0.27 \pm 0.03$ & $-0.99 \pm 0.02$ & $-0.99 \pm 0.02$ \\
north & 53 & 236 & $0.22 \pm 0.03$ & $-0.93 \pm 0.03$ & $-0.95 \pm 0.02$ \\
south & 88 & 286 & $0.31 \pm 0.04$ & $-1.02 \pm 0.03$ & $-1.02 \pm 0.02$ \\
\hline
\end{tabular}

${ }^{a}$ Calculated from the correlation of Battinelli \& Demers (2005a).

${ }^{b}$ Calculated from the correlation of Cioni \& Habing (2005).

C-rich stars, i.e. C stars at lower metallicity, and the AGB evolution models by Marigo et al. (1999) predict that the third dredgeup, which brings carbon and s-process elements to the stellar surface, occurs widely as metallicity decreases. Battinelli \& Demers (2005a) presented updated C/M ratios for the LG galaxies and showed that the $\mathrm{C} / \mathrm{M}$ ratio correlates well with the metallicity of the parent galaxy, i.e. $[\mathrm{Fe} / \mathrm{H}]=-1.32-0.59 \times \log (\mathrm{C} / \mathrm{M})$. Cioni $\&$ Habing (2005) obtained a calibration of the relation between $\mathrm{C} / \mathrm{M}$ and $[\mathrm{Fe} / \mathrm{H}]$ as $[\mathrm{Fe} / \mathrm{H}]=-1.23-0.42 \times \log (\mathrm{C} / \mathrm{M})$ using the dataset of Groenewegen $(2004,2002)$ for the $\mathrm{C} / \mathrm{M}$ and $[\mathrm{Fe} / \mathrm{H}]$ of the LG galaxies. Applying these relations, we calculated $[\mathrm{Fe} / \mathrm{H}] \approx-0.99$ in the total observed area of NGC 6822 with $[\mathrm{Fe} / \mathrm{H}] \sim-1.02$ in the southern bar and $[\mathrm{Fe} / \mathrm{H}]=-0.93 \sim-0.95$ in the northern bar, which is similar to the mean metallicity $[\mathrm{Fe} / \mathrm{H}]=-0.9$ of RGB stars in the northern part of the bar derived by Tolstoy (2001). The result indicates a small difference in mean metallicities $\Delta[\mathrm{Fe} / \mathrm{H}] \approx 0.07 \sim 0.09$ dex between the northern and southern parts in the central bar of NGC 6822. Table 4 summarizes the number of detected $\mathrm{C}$ stars and $\mathrm{M}$-giants, $\mathrm{C} / \mathrm{M}$ ratio, and the derived metallicity in the total observed area, and the northern and southern parts in the central bar region of NGC 6822 .

\subsection{Color distributions}

The range of ages for AGB stars in the central bar of NGC 6822 is inferred by comparing the color distributions in $(J-K)$ and $(H-K)$ with the theoretical evolutionary models of
AGB stars (Girardi et al. 2002). The upper panels of Fig. 9 show the histogram distributions of the $(J-K)$ (left) and $(H-K)$ (right) colors for selected 663 AGB stars in NGC 6822. It is apparent that both $(J-K)$ and $(H-K)$ histograms have an M-giant peak, a red tail of $\mathrm{C}$ stars, and a blue excess. The shapes of the partial color distributions in the southern and northern parts of the bar do not show significantly different features from the color histograms for all selected AGB stars.

Eye-fitted Gaussians are traced over the histograms with $\sigma$ values equal to the random photometric errors of $\sigma_{(J-K)}=$ 0.064 and $\sigma_{(H-K)}=0.058$ predicted from artificial star experiments (Fig. 2). The red tails correspond to the identified C stars, which produce an excess population of objects with respect to M-giants where $(J-K)>1.53$ and $(H-K)>0.50$ as defined in Sect. 3.3. The blue excesses are also shown in the color histograms where $(J-K) \lesssim 1.15$ and $(H-K) \lesssim 0.25$, due to younger AGB stars than those along the M-giant peaks and possibly a few supergiant stars remaining after the AGB star selection (cf. Sect. 3.3).

In our previous papers (Kang et al. 2005; Sohn et al. 2006), we used the color histograms of NGC 185 and NGC 147 to estimate the range of ages for intermediate age populations in each galaxy. Kang et al. (2005) estimated two possible epochs of star formation for intermediate age populations in NGC 185 with $\log \left(t_{\mathrm{yr}}\right) \approx 9.0 \sim 9.4$ and $7.8 \sim 8.5$, and Sohn et al. (2006) estimated a range of star-formation epochs in NGC 147 with $\sim 8.2 \lesssim$ $\log \left(t_{\mathrm{yr}}\right) \lesssim 8.6$ having a peak of $\log \left(t_{\mathrm{yr}}\right)=8.4$. Meanwhile, Davidge (2005) inferred the range of ages for M-giant stars in NGC 147, NGC 185, and NGC 205 by comparing the $(J-K)$ and $(H-K)$ colors on the color histograms of M-giant stars of $-7.6<M_{K}<-7.2$ with those derived from the theoretical isochrones of M-giant stars at $M_{K}=-7.4$ for $\log \left(t_{\mathrm{yr}}\right)=8.1$, 9.0, and 9.8 (Girardi et al. 2002). To determine the range of ages for M-giant stars in NGC 6822, we also applied the same technique to the isochrones of AGB stars, with $Z=0.004$. The lower panels of Fig. 9 show the histogram distributions of the $(J-K)($ left $)$ and $(H-K)$ (right) colors for $78 \mathrm{M}$-giant stars with $M_{K}$ between -7.2 and -7.6 per 0.1 mag interval. Arrows are the theoretically predicted colors at $M_{K}=-7.4$ for ages of $\log \left(t_{\mathrm{yr}}\right)=7.8,8.7$, and 10.2 with the adopted distance and reddening of NGC 6822 . The broad color distributions for M-giants indicate that NGC 6822 contains M-giants with a wide range of intermediate ages $\log \left(t_{\mathrm{yr}}\right) \approx 8 \sim 10$ having the peak of $\log \left(t_{\mathrm{yr}}\right) \sim 9.0$.

\subsection{Luminosity functions}

The luminosity function (LF) for the resolved AGB stars in a galaxy is one of the most important concepts used to compare actual observations with evolutionary models of M-giants of AGB population and C stars. Indeed, bolometric LF and the mean bolometric magnitude of $\mathrm{C}$ stars in a galaxy depend on metallicity and the star formation history of a galaxy (Nowotny et al. 2003; Groenewegen 1999). Battinelli et al. (2003) suggest that the $i$ band LF of AGB stars can be used as a standard candle to estimate the distance of galaxies. It is worth noting that 


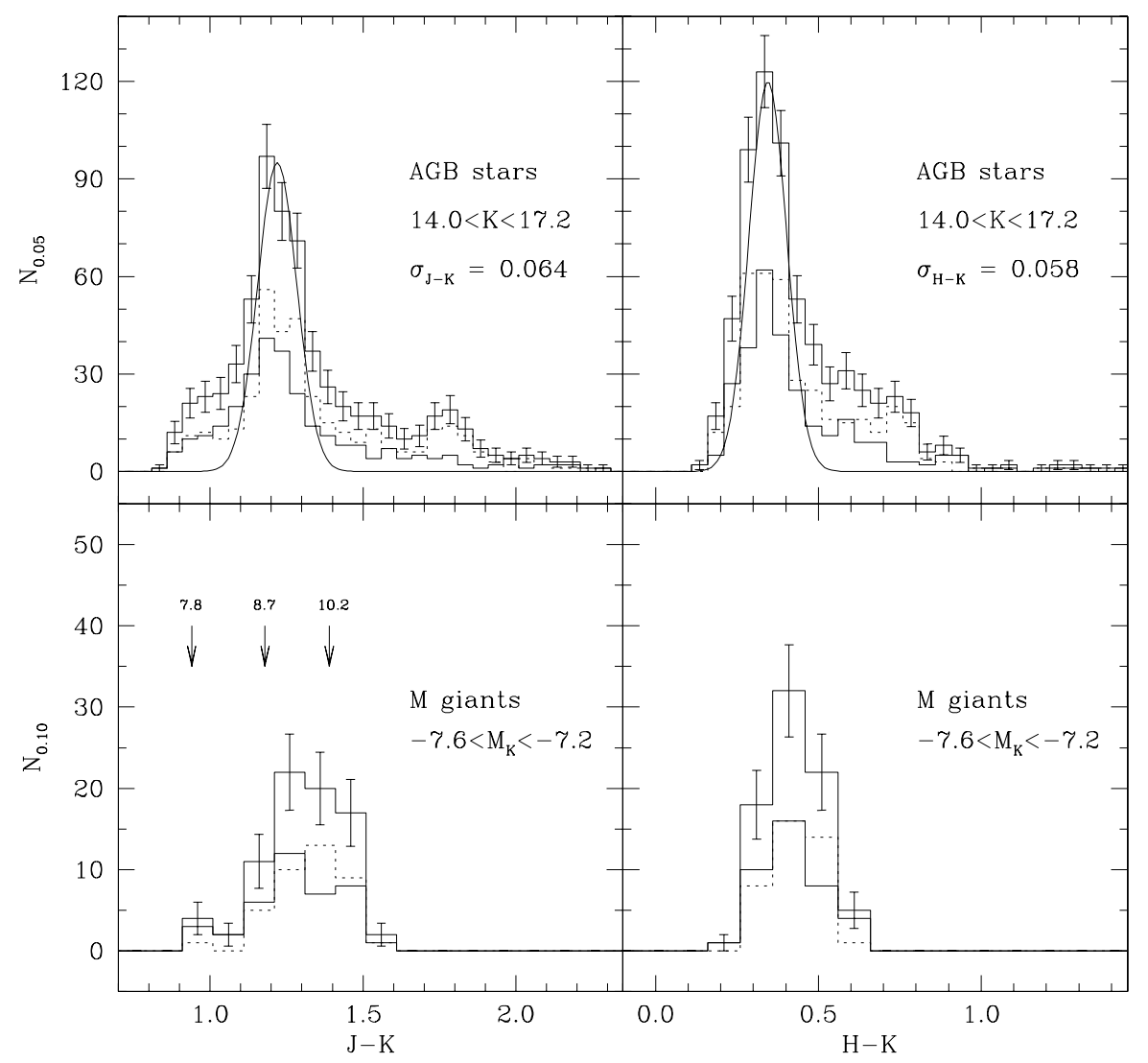

Fig. 9. Upper: the histogram distributions of $(J-K)(l e f t)$ and $(H-K)(r i g h t)$ colors for selected 663 AGB stars in NGC 6822 . The solid and dotted lines without error bars show color histograms of AGB stars at the northern and southern parts in the bar of NGC 6822, respectively. Lower: the histogram distributions of colors for $78 \mathrm{M}$-giant stars with $M_{K}$ between -7.2 and -7.6 per 0.1 mag interval in color. Arrows indicate the theoretically predicted $(J-K)$ colors of the isochrones for M-giant stars (Girardi et al. 2002) at $M_{K}=-7.4$ with $Z=0.004$ and ages of $\log \left(t_{\mathrm{yr}}\right)=7.8,8.7$ and 10.2 .

near-infrared observations have an advantage over visible band observations in deriving the LFs of AGB stars in a galaxy, as Freedman (1992) noted that some very red bright stars which are visible in near-infrared images are not always detected in optical band images. Figure 10 shows the completeness-corrected LFs for M-giants and C stars detected in the central bar of NGC 6822.

The two diagrams on the left of Fig. 10 represent the LFs in $M_{K}$ for M-giants and $\mathrm{C}$ stars detected in the whole observed area of NGC 6822 and for those of the northern and southern bars. The LF of C stars in $M_{K}$ is likely to be a Gaussian distribution. A two-sided Kolmogorov-Smirnov test of the LF and the Gaussian distribution of the mean absolute magnitude and standard deviation $\left\langle M_{K}\right\rangle=-7.60 \pm 0.50$, which were estimated in Sect. 4.1, show to a $94 \%$ confidence level that the distribution between the two is the same. We note that most of the $I$ band LFs of $C$ stars in nearby dwarf galaxies also fit a Gaussian distribution (Battinelli \& Demers 2004a,b). It is apparent that the LF of $\mathrm{C}$ stars in the southern part is skewed to a brighter side than in the northern part by $M_{K} \sim 0.25 \mathrm{mag}$, while M-giant stars do not show significantly different shapes of LFs in either region. The different environmental conditions for star formation and evolution would affect the different absolute magnitudes of $\mathrm{C}$ stars. From the relation between the mean absolute I magnitude of $\mathrm{C}$ stars and the metallicity of the parent galaxy, Battinelli \& Demers (2005b) confirm that metal-poor AGB stars turn to $\mathrm{C}$ stars sooner and reach brighter luminosity as a consequence of less effective mass loss. In Sect. 4.1, we showed that the mean metallicity of AGB stars in the southern part is lower than in the northern part by a small difference of $\Delta[\mathrm{Fe} / \mathrm{H}] \approx 0.07 \sim 0.09 \mathrm{dex}$. Here, we cautiously attribute the skewed LF in $M_{K}$ of $\mathrm{C}$ stars in the southern part of the bar to the metal-poor environment, which may lead to slightly bluer mean colors of the AGB and RGB stars in the southern part than those in the northern part.

To examine the differences in color, we estimated the mean colors of the selected AGB stars and the RGB stars $(17.2<K \leq$ $20.2)$ in the southern and northern parts of the bar. The results are listed in Table 5. The mean colors of the RGB stars in the southern part seem to be slightly bluer than those in the northern part with small confidence levels of less than $1 \sigma$ standard error, while the mean colors of the resolved AGB stars in the southern part are nearly identical with those in the northern part. We caution, however, that the estimated mean colors of the AGB and RGB stars do not have a strong confidence to infer the metallicity difference between the southern and northern part of the bar, because of the small number of resolved AGB stars and the incomplete RGB sample with some main-sequence stars and field stars.

The small inner panel in the lower-left diagram of Fig. 10 shows the completeness-corrected logarithmic LF in $M_{K}$ of M-giants detected in the observed area of NGC 6822. By performing a least-square fit to the brightness interval of $-8.7<$ $M_{K}<-7.1$, where the completeness is still high, we estimated the exponent of the $M_{K}$ LF for the bright M-giants in NGC 6822 to be $1.12 \pm 0.03$. Comparing the slopes for nearby dwarf elliptical galaxies, i.e. $0.83 \pm 0.02$ for NGC 185 (Kang et al. 2005) 


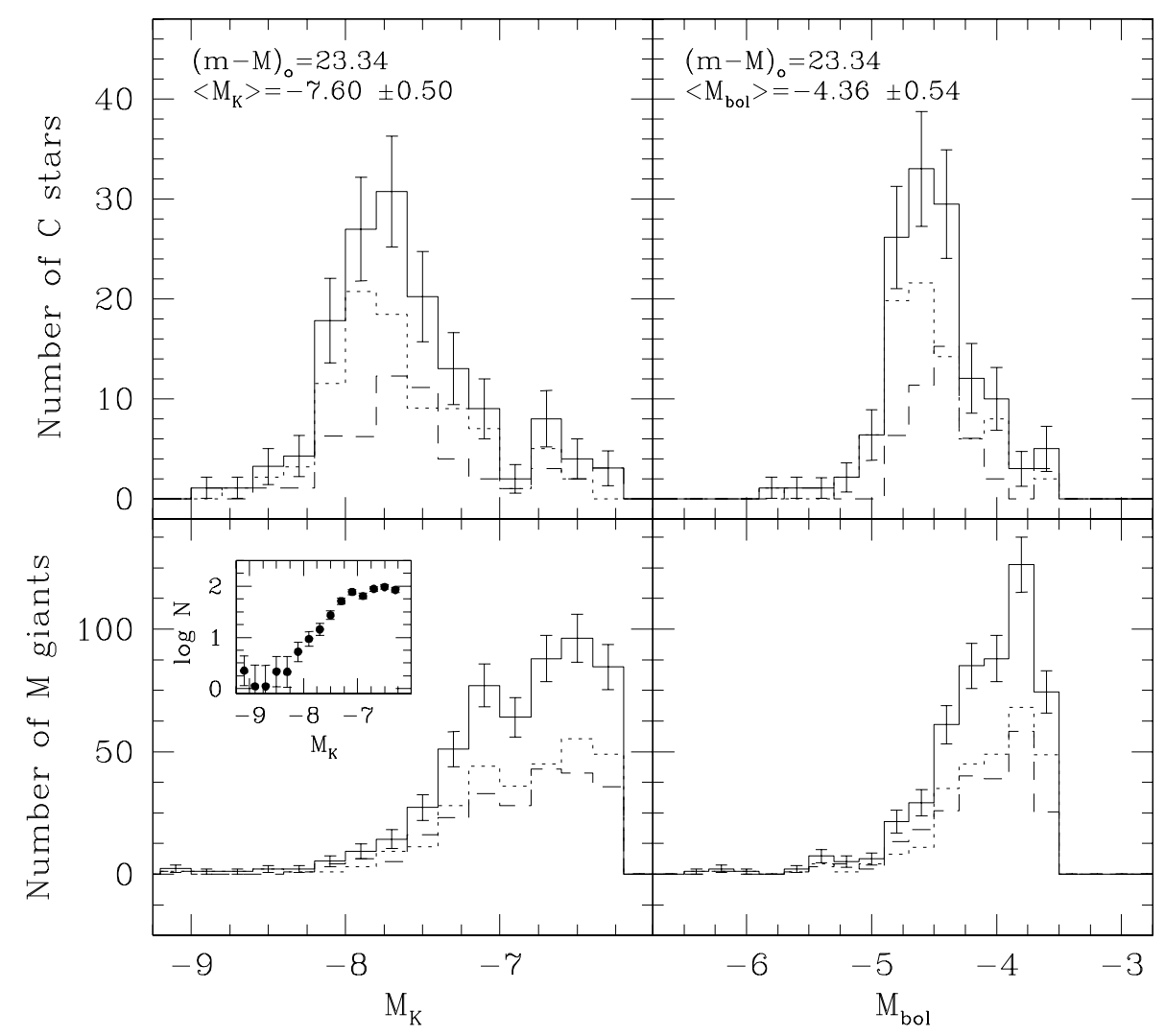

Fig. 10. Left: the completeness-corrected LFs in $M_{K}$ for M-giants and C stars. The inner small panel in the lower panel shows the logarithmic LF of M-giant in NGC 6822. The LFs with solid lines represent M-giants and C stars detected in the whole observed area of NGC 6822. The LFs with dashed and dotted lines are those of the northern bar and southern bars of NGC 6822, respectively. Right: the completeness-corrected bolometric LFs for M-giant stars and C stars. The different line types are the same as in the left hand diagrams.

Table 5. The mean colors of the selected AGB stars and the RGB stars in the bar of NGC 6822 .

\begin{tabular}{lccc}
\hline \hline Population & Position & $\overline{(J-K)}$ & $\overline{(H-K)}$ \\
\hline AGB & south & $1.36 \pm 0.34$ & $0.45 \pm 0.20$ \\
& north & $1.34 \pm 0.40$ & $0.46 \pm 0.27$ \\
RGB $(17.2<K \leq 20.2)$ & south & $0.91 \pm 0.37$ & $0.26 \pm 0.31$ \\
& north & $1.00 \pm 0.36$ & $0.29 \pm 0.35$ \\
\hline
\end{tabular}

and $0.79 \pm 0.02$ for NGC 147 (Sohn et al. 2006), the LF in $M_{K}$ of M-giant stars in the dwarf irregular galaxy NGC 6822 seems to have a larger slope. This result implies that the star-forming history for M-giants in the dwarf irregular galaxy NGC 6822 (e.g. Wyder 2003, 2001) is likely to be different from those in dwarf elliptical galaxies. More near-infrared photometry of the resolved AGB stars in nearby galaxies is needed to check whether the slope in the $K$ band LF of M-giants depends on the star-formation conditions of the different galaxy's morphologies.

The bolometric corrections in $K$ band, $B C_{K}$, of the M-giants, and $\mathrm{C}$ stars in the central bar of NGC 6822 were estimated from the empirical relations between $B C_{K}$ and $(J-K)$ for Galactic and LMC AGB stars. The relations for the Galactic and LMC M-giant stars obtained by Bessell \& Wood (1984) were applied to the M-giants, while the relation for the Galactic C stars given by Costa \& Frogel (1996) was applied to C stars in NGC 6822. The two diagrams to the right in Fig. 10 show the completeness corrected bolometric LFs of M-giant and C stars in the central bar of NGC 6822. For the lower limit, we followed the usual $M_{\text {bol }}<-3.5$ threshold corresponding to the bolometric magnitude of the tip of RGB (Ferraro et al. 2000). As in the LFs of $M_{K}$, the bolometric LF of C stars in the southern part is also skewed to the brighter side when compared with the northern part, while M-giant stars do not show significantly different shapes of bolometric LFs in either region. The bolometric LF for M-giant stars in NGC 6822 extends up to $M_{\mathrm{bol}}=-6.5 \mathrm{mag}$, and that of C stars spans $-5.8<M_{\mathrm{bol}}<-3.5$. The mean bolometric magnitude of C stars in NGC 6822 is estimated to be $M_{\text {bol }}=-4.36 \pm 0.54$, which is comparable to those of C stars in NGC 147 and NGC 185, i.e. $\left\langle M_{\text {bol }}\right\rangle=-4.32 \pm 0.49$ for NGC 147 (Sohn et al. 2006) and $\left\langle M_{\mathrm{bol}}\right\rangle=-4.50 \pm 0.42$ for NGC 185 (Kang et al. 2005), respectively.

\section{Conclusion}

The $g i$ and $J H K$ images were used to investigate the bright stellar contents of the AGB population in the central bar of the nearby dwarf irregular galaxy NGC 6822. The results are as follows:

1. The CMDs of NGC 6822 based on the gi and JHK photometry show populations of main-sequence stars, foreground stars, massive supergiants, RGB, and AGB stars. The AGB population in the CMDs contains a group of dominant M-giant stars and red C stars.

2 . In the $(g-K, g) \mathrm{CMD}$, which has a long wavelength baseline color index, AGB stars are well distinguished from the other bright young stellar populations, such as supergiant stars and foreground stars. A comparison of theoretical isochrones of AGB stars with the observed $(J-K, K)$ CMD indicates that most AGB stars are distributed in the range of 
ages $\log \left(t_{\mathrm{yr}}\right) \sim 8.7-10.2$, which suggests that the star formation in the central bar of NGC 6822 has been continuous for intermediate age.

3. A total of $141 \mathrm{C}$ stars are separated from M-giant stars in the near-infrared color-color diagram. The mean magnitude and colors of the $141 \mathrm{C}$ stars in the central bar of NGC 6822 are estimated to be $\langle K\rangle=15.82 \pm 0.50,\langle(J-K)\rangle=1.90 \pm 0.41$, and $\langle(H-K)\rangle=0.80 \pm 0.28$, which correspond to $\left\langle M_{K}\right\rangle=$ $-7.60,\left\langle(J-K)_{0}\right\rangle=1.77$, and $\left\langle(H-K)_{0}\right\rangle=0.75$ at the distance of NGC 6822. The mean magnitude and colors of $\mathrm{C}$ stars are not significantly different through the observed central bar region.

4. The $\mathrm{C} / \mathrm{M}$ ratio at the total observed area is estimated to be $0.27 \pm 0.03$, while the northern part of the bar has a rather lower $\mathrm{C} / \mathrm{M}$ ratio than the southern part of the bar. The correlation between the $\mathrm{C} / \mathrm{M}$ ratio and metallicity used to derive a small spread of metallicities $\Delta[\mathrm{Fe} / \mathrm{H}] \approx 0.07 \sim 0.09$ dex in the bar.

5. The $(J-K)$ and $(H-K)$ color distributions of AGB stars contain a main peak with M-giant stars, a red tail with $\mathrm{C}$ stars, and a blue excess with supergiants. The broad color distributions for M-giants indicate that NGC 6822 has a wide range of intermediate ages with a peak at $\log \left(t_{\mathrm{yr}}\right) \sim 9.0$.

6. The $M_{K} \mathrm{LF}$ for C stars in the central bar of NGC 6822 is likely to be a Gaussian distribution, while the LF of C stars in the southern part is slightly skewed to the brighter side than in the northern part by $\Delta M_{K} \sim 0.25 \mathrm{mag}$.

7. The slope of the logarithmic $K$ band LF of M-giant stars is estimated to be $1.12 \pm 0.03$.

8. In the bolometric LFs, M-giants extend up to $M_{\text {bol }}=-6.5$ and $\mathrm{C}$ stars spans $-5.8<M_{\text {bol }}<-3.5$. The mean bolometric magnitude of C stars in NGC 6822 is estimated to be $M_{\mathrm{bol}}=$ $-4.36 \pm 0.54$.

Acknowledgements. We thank the referee P. Battinelli for his useful comments that greatly improved this paper. Support for this work was provided by Korea Astronomy and Space Science Institute, for which we are grateful. M.G.L. is in part supported by the KOSEF grant (R01-2004-000-10490-0). Y.C.K. is supported by the Astrophysical Center for the Structure and Evolution of the Cosmos (ARCSEC) of the Korea Science and Engineering Foundation (KOSEF) through the Science Research Center (SRC) Program.

\section{References}

Battinelli, P., \& Demers, S. 2004a, A\&A, 417, 479

Battinelli, P., \& Demers, S. 2004b, A\&A, 418, 33

Battinelli, P., \& Demers, S. 2005a, A\&A, 434, 657

Battinelli, P., \& Demers, S. 2005b, A\&A, 442, 159

Battinelli, P., Demers, S., \& Letarte, B. 2003, AJ, 125, 1298

Bessell, M. S., \& Wood, P. R. 1984, PASP, 96, 247

Cioni, M.-R. L., \& Habing, H. J. 2005, A\&A, 429, 837

Cioni, M.-R. L., Habing, H. J., \& Israel, F. P. 2000a, A\&A, 358, L9

Cioni, M.-R. L., van der Marel, R. P., Loup, C., \& Habing, H. J. 2000b, A\&A, 359,601

Cohen, J. G., \& Blakeslee, J. P. 1998, AJ, 115, 2356

Cohen, J. G., Persson, S. E., \& Frogel, J. A. 1978, ApJ, 222, 165

Cook, K. H., Aaronson, M., \& Norris, J. 1986, ApJ, 305, 634

Costa, E., \& Frogel, J. A. 1996, AJ, 112, 2607

Davidge, T. J. 2003a, ApJ, 597, 289

Davidge, T. J. 2003b, PASP, 115, 635

Davidge, T. J. 2005, AJ, 130, 2087

de Blok, W. J. G., \& Walter, F. 2000, ApJ, 537, L95

Demers, S., Battinelli, P., \& Kunkel, W. E. 2005, ApJ, in print

Ferraro, F. R., Montegriffo, P., Origlia, L., \& Fusi Pecci, F. 2000, AJ, 119, 1282

Freedman, W. D. 1992, AJ, 104, 1349

Frogel, J. A., Persson, S. E., \& Cohen, J. G. 1980, ApJ, 239, 495

Gallart, C., Aparicio, A., Chiosi, C., Bertelli, G., \& Vilches, J. M. 1994, ApJ, 425, L9

Gallart, C., Aparicio, A., Bertelli, G., \& Chiosi, C. 1996a, AJ, 112, 1950
Gallart, C., Aparicio, A., Bertelli, G., \& Chiosi, C. 1996b, AJ, 112, 2596

Gallart, C., Aparicio, A., \& Vilchez, J. M. 1996c, AJ, 112, 1928

Girardi, L., Bertelli, G., Bressan, A., et al. 2002, A\&A, 391, 195

Gottesman, S. T., \& Weliachew, L. 1977, A\&A, 61, 523

Grebel, E. K. 1997, Rev. Mod. Astron., 10, 29

Grebel, E. K. 1999, in The Stellar Content of the Local Group, ed. P. Whitelock, \& R. Cannon (San Francisco: ASP), IAU Symp., 192, 17

Grebel, E. K. 2000, ASPC, 221, 297

Grebel, E. K. 2001, Ap\&SS, 277, 231

Grebel, E. K. 2005, AIPC, 752, 161

Groenewegen, M. A. T. 1999, in Asymptotic Giant Branch Stars, ed. T. Le Berte, A. Lèbre, \& C. Waelkens (San Francisco: ASP), IAU Symp., 191, 535

Groenewegen, M. A. T. 2002, in Chemical Evolution of Dwarf Galaxies, Ringberg Castle Workshop [arXiv: astro-ph/0208449]

Groenewegen, M. A. T. 2004, in Planetary Nebulae beyond the Milky Way, Proc. of ESO Workshop, ed. J. Walsh, L. Stanghellini, \& N. Douglas (Garching: ESO), in press [arXiv: astrp-ph/0407282]

Guarnieri, M. D., Ortolani, S., Montegriffo, P., et al. 1998, A\&A, 331, 70

Hodge, P. W. 1977, ApJS, 33, 69

Hodge, P. W., Kennicutt, R. C., \& Lee, M. G. 1988, PASP, 100, 917

Hodge, P. W., Smith, T., Eskridge, P., MacGillivray, H., \& Beard, S. 1991, ApJ, 379,621

Holtzman, J. A., Afonso, C., \& Dolphin, A. 2002, A\&AS, 200, 4603

Hutchings, J. B., Cavanagh, B., \& Bianchi, L. 1999, PASP, 111, 559

Hwang, N., Lee, M. G., Lee, J. C., et al. 2005, in the Near-Field Cosmology with Dwarf Elliptical Galaxies, ed. H. Jerjen, \& B. Binggeli (Cambridge: Cambridge University Press), IAU Coll., 198, 257

Iben, I. Jr., \& Renzini, A. 1983, ARA\&A, 21, 271

Kang, A., Sohn, Y.-J., Rhee, J., et al. 2005, A\&A, 437, 61

Komiyama, Y., Okamura, S., Masafumi, Y., et al. 2003, ApJ, 590, L17

Kuchinski, L. E., Frogel, J. A., Terndrup, D. M., \& Persson, S. E. 1995, AJ, 109, 1131

Lee, M. G., \& Hwang, N. 2005, in the Near-Field Cosmology with Dwarf Elliptical Galaxies, ed. H. Jerjen, \& B. Binggeli (Cambridge: Cambridge University Press), IAU Coll., 198, 181

Lee, M. G., Freedman, W. L., \& Madore, B. F. 1993, ApJ, 417, 553

Letarte, B., Demers, S., Battinelli, P., \& Kunkel, W. E. 2002, AJ, 123, 832

Marigo, P., Girardi, L., \& Bressan, A. 1999, A\&A, 344, 123

Marigo, P., Girardi, L., \& Chiosi, C. 2003, A\&A, 403, 225

Massey, P., Armandroff, T. E., Pyke, R., Patel, K., \& Wilson, C. D. 1995, AJ, 110,2715

Mateo, M. 1998, ARA\&A, 36, 435

McAlary, C. W., Madore, B. F., McGonegal, R., Mclaren, R. A., \& Welch, D. L. 1983, ApJ, 273, 539

Momany, Y., Ortolani, S., Held, E. V., et al. 2003, A\&A, 402, 607

Nowotny, W., Kerschbaum, F., Olofsson, H., \& Schwarz, H. E. 2003, A\&A, 403, 93

Nowotny, W., Kerschbaum, F., Schwarz, H. E., \& Olofsson, H. 2001, A\&A, 367, 557

O’Dell, C. R., Hodge, P. W., \& Kennicutt, R. C. 1999, PASP, 111, 1382

Pietrzyński, G., Gieren, W., Udalski, A., et al. 2004, AJ, 128, 2815

Ratnatunga, K. U., \& Bahcall, J. N. 1985, ApJS, 59, 63

Rizzi, L. Held, E. V., Momany, Y., et al. 2003, Mem. Soc. Astron. Ital., 74, 510

Roberts, M. S. 1972, in External Galaxies and Quasi-stellar Objects, ed. D. S. Evans (Dordrecht: Reidel), IAU Symp., 44, 12

Schlegel, D. J., Finkbeiner, D. P., \& Davis, M. 1998, ApJ, 500, 525

Simons, D. A., \& Tokunaga, A. 2002, PASP, 114, 169

Sohn, Y.-J., Kang, A., Rhee, J., et al. 2006, A\&A, 445, 69

Stetson, P. B. 1987, PASP, 99, 191

Stetson, P. B. 1994, PASP, 106, 250

Stetson, P. B., \& Harris, W. E. 1988, AJ, 96, 909

Tokunaga, A. T., Simons, D. A., \& Vacca, W. D. 2002, PASP, 114, 180

Tolstoy, E. 2003, Ap\&SS, 284, 579

Tolstoy, E., Irwin, M. J., Cole, A. A., et al. 2001, MNRAS, 327, 918

Valenti, E., Ferraro, F. R., \& Origlia, L. 2004, MNRAS, 354, 815

van den Bergh, S. 1999, A\&ARv, 9, 273

van den Bergh, S. 2000, The Galaxies of the Local Group, Cambridge Astrophys. Ser., 35

Venn, K. A., Lennon, D. J., Kaufer, A., et al. 2001, ApJ, 547, 765

Visvanathan, N. 1989, ApJ, 346, 629

Volders, L., \& Högbom, J. A. 1961, Bull. Astron. Inst. Netherlands, 15, 307

Wainscoat, R. J., \& Cowie, L. L. 1992, AJ, 103, 332

Weldrake, D. T. F., de Blok, W. J. G., \& Walter, F. 2003, MNRAS, 340, 12

Wilson, C. D. 1992a, AJ, 104, 1374

Wilson, C. D. 1992b, ApJ, 384, L29

Wood, P. R., Bessell, M. S., \& Paltoglou, G. 1985, ApJ, 290, 477

Wyder, T. K. 2001, AJ, 122, 2490

Wyder, T. K. 2003, AJ, 125, 3097 